\title{
Fisher information, Wehrl entropy, and Landau diamagnetism
}

\author{
S. Curilef, ${ }^{1}$ F. Pennini, ${ }^{2}$ and A. Plastino ${ }^{2}$ \\ ${ }^{1}$ Departamento de Física, Universidad Católica del Norte, Av. Angamos 0610, Antofagasta, Chile \\ ${ }^{2}$ Instituto de Física La Plata (IFLP), Universidad Nacional de La Plata (UNLP), \\ and Argentine National Research Council (CONICET), C.C. 727, 1900 La Plata, Argentina \\ (Received 7 September 2004; revised manuscript received 7 October 2004; published 25 January 2005)
}

\begin{abstract}
Using information theoretic quantities like the Wehrl entropy and Fisher's information measure we study the thermodynamics of the problem leading to Landau's diamagnetism, namely, a free spinless electron in a uniform magnetic field. We reveal a new Fisher-uncertainty relation, derived via the Cramer-Rao inequality, that involves phase space localization and energy fluctuations.
\end{abstract}

DOI: 10.1103/PhysRevB.71.024420

PACS number(s): 75.20.- $\mathrm{g}, 03.65 . \mathrm{Sq}, 05.70 .-\mathrm{a}, 03.67 .-\mathrm{a}$

\section{INTRODUCTION}

The last years have witnessed a great deal of activity revolving around physical applications of Fisher's information measure (FIM) $I$ (as a rather small sample, see for instance, Ref. 1-5). Frieden and Soffer ${ }^{1}$ have shown that Fisher's information measure provides one with a powerful variational principle, the extreme physical information one, that yields most of the canonical Lagrangians of theoretical physics. ${ }^{1,2}$ Additionally, $I$ has been shown to provide an interesting characterization of the "arrow of time," alternative to the one associated with Boltzmann's entropy. ${ }^{6,7}$ Also to be emphasized is the fact that both equilibrium and off-equilibrium thermodynamics can be entirely based upon Fisher's measure, a procedure that exhibits definite advantages over conventional textbook treatments. ${ }^{8}$

Unraveling the multiple FIM facets and their links to physics should be of general interest to a vast audience. Our present subject matter is the information theory treatment of Landau-diamagnetism's thermodynamics, that we will study using two information measures, namely FIM and a special instance of Shannon's logarithmic one, called the Wehrl entropy $W$.

$W$ is a very useful measure of localization in phase space. ${ }^{9,10}$ It is built up using coherent states ${ }^{9,11,12}$ and constitutes a powerful tool in statistical physics. The pertinent definition reads

$$
W=-\int \frac{\mathrm{d} x \mathrm{~d} p}{2 \pi \hbar} \mu(x, p) \ln \mu(x, p),
$$

where $\mu(x, p)=\langle z|\rho| z\rangle$ is a "semiclassical" phase-space distribution function associated to the density matrix $\rho$ of the system, ${ }^{12-14}$ often referred to as the Husimi distribution. ${ }^{15}$ The distribution $\mu(x, p)$ is normalized in the fashion $\int(\mathrm{d} x \mathrm{~d} p / 2 \pi \hbar) \mu(x, p)=1$. Indeed, $\mu(x, p)$ is a Wignerdistribution $D_{W}$ smeared over an $\hbar$ sized region of phase space. ${ }^{11}$ The smearing renders $\mu(x, p)$ a positive function, even if $D_{W}$ does not have such a character. The semiclassical Husimi probability distribution refers to a special type of probability: that for simultaneous but approximate location of position and momentum in phase space. ${ }^{11}$ The uncertainty principle manifests itself through the inequality

$$
W \geqslant 1,
$$

which was first conjectured by Wehrl ${ }^{9}$ and later proved by Lieb. ${ }^{16}$ Equality holds iff $\rho$ is a coherent state. ${ }^{9,16}$ This scenario will have to be modified in the case of Landau's diamagnetism problem, as we will show below.

The usual treatment of equilibrium in statistical mechanics makes use of the Gibbs's canonical distribution, whose associated, "thermal" density matrix is given by $\rho=Z^{-1} e^{-\beta \mathcal{H}}$, with $Z=\operatorname{Tr}\left(e^{-\beta \mathcal{H}}\right)$ the partition function, $\beta=1 / k T$ the inverse temperature $(T)$, and $k$ the Boltzmann constant. In order to conveniently write down an expression for $W$ one considers, for the Hamiltonian $\mathcal{H}$, its eigenstates $|n\rangle$ and eigenenergies $E_{n}$, because one can always write ${ }^{11}$

$$
\mu(x, p)=\langle z|\rho| z\rangle=\frac{1}{Z} \sum_{n} e^{-\beta E_{n}}|\langle z \mid n\rangle|^{2} .
$$

A useful route to $W$ starts then with (3) and continues with (1).

Quantum-mechanical phase-space distributions expressed in terms of the phase states $|z\rangle$ of the harmonic oscillator have been proved to be useful in different contexts. ${ }^{12-14}$ Particular reference is made to the illuminating work of Andersen and Halliwell, ${ }^{11}$ who discuss, among other things, the concepts of Husimi distributions and Wehrl entropy. Coherent states are eigenstates of a general annihilation operator $a$, appropriate for the problem at hand, i.e., $a|z\rangle=z|z\rangle .{ }^{12-14} \mathrm{In}$ the special case of the harmonic oscillator whose Hamiltonian has the form $\mathcal{H}_{o}=\hbar \omega\left[a^{\dagger} a+1 / 2\right]$, the complex plane of eigenvalues of the annihilation operator $a$ are given by

$$
z=(m \omega / 2 \hbar)^{1 / 2} x+i(2 \hbar \omega m)^{-1 / 2} p .
$$

In this case, a new relation between the entropy $W$ and the measure $I$ of the equilibrium thermal state was found in Ref. 19. Accordingly, these two measures become complementary informational quantities and satisfy the condition $W+\ln I$ $=1$. A natural question that we shall address here is the following: will the relation of Ref. 19 retain its form for Landau's diamagnetism? The answer is no, which makes the thermodynamics of the latter problem definitely different to that of the harmonic oscillator. Notice that, in this instance, (i) 
$W \geqslant 1$ implies $I \leqslant 1$ and (ii) the use of coherent states maximizes the Fisher measure.

For the convenience of the reader this paper is organized as follows: in Sec. II we summarize important aspects of constructing a complete set of coherent states for a spinless electron in a uniform magnetic field. This enables one to obtain the pertinent Husimi distribution in phase-space given [cf. Eq. (3)] in Sec. III, where we explicitly evaluate it, together with concomitant Wehrl entropy. We introduce in Sec. IV basic notions regarding Fisher's information measure (FIM). In Sec. V we construct the appropriate FIM in phasespace one for Landau's diamagnetism, and finally, we draw some conclusions in Sec. VI.

\section{ELECTRON IN A UNIFORM MAGNETIC FIELD}

We enter the present application by revisiting the complete set of coherent states of a spinless electron in a uniform magnetic field, generated by using the step-ladder operator. ${ }^{17}$ Consider the kinetic momentum

$$
\vec{\pi}=\vec{p}+\frac{e}{c} \vec{A}
$$

of a particle of charge $e$, mass $m_{r}$, and linear momentum $\vec{p}$, subject to the action of a vector potential $\vec{A}$. These are the essential ingredients of the well-known Landau model for diamagnetism, a spinless electron in a magnetic field of intensity $H$ (we follow the presentation of Feldman et al. ${ }^{17}$ ). The Hamiltonian reads ${ }^{17}$

$$
\mathcal{H}=\frac{\vec{\pi} \cdot \vec{\pi}}{2 m_{r}}
$$

and the magnetic field is $\vec{H}=\vec{\nabla} \times \vec{A}$. The vector potential is chosen in the symmetric gauge as $\vec{A}=(-H y / 2, H x / 2,0)$, which corresponds to a uniform magnetic field along the $z$ direction. One also needs the step operators ${ }^{17}$

$$
\pi_{ \pm}=p_{x} \pm i p_{y} \pm \frac{i \hbar}{2 l^{2}}(x \pm i y)
$$

Motion along the $z$ axis is free. ${ }^{17}$ For the transverse motion, the Hamiltonian specializes to ${ }^{17}$

$$
\mathcal{H}_{t}=\frac{\pi_{+} \pi_{-}}{2 m_{r}}+\frac{1}{2} \hbar \Omega \text {. }
$$

Two important quantities characterize the problem, namely, $\Omega=e H / m_{r} c$, the cyclotron frequency and the length $l$ $=(\hbar c / e H)^{1 / 2} \cdot{ }^{18}$ The eigenstates $|N, m\rangle$ are determined by two quantum numbers, $N$ (associated to the energy), and $m$ (to the $z$ projection of the angular momentum). As a consequence, they are simultaneously eigenstates of both $\mathcal{H}_{t}$ and the angular momentum operator $L_{z},{ }^{17}$ so that

$$
\mathcal{H}_{t}|N, m\rangle=\left(N+\frac{1}{2}\right) \hbar \Omega|N, m\rangle=E_{N}|N, m\rangle
$$

and

$$
L_{z}|N, m\rangle=m \hbar|N, m\rangle \text {. }
$$

We note that the eigenvalues of $L_{z}$ are not bounded by below ( $m$ takes the values $-\infty, \ldots,-1,0,1, \ldots, N) .{ }^{17}$ This agrees with the fact that the energies $(N+1 / 2) \hbar \Omega$ are infinitely degenerate. ${ }^{18}$ Such a fact diminishes the physical relevance of phase-space localization for estimation purposes, as we shall see below. Moreover, $L_{z}$ is not an independent constant of the motion. ${ }^{18}$

We face here a bi-dimensional phase-space problem. The corresponding four phase-space variables can conveniently be called $x, y, p_{x}$, and $p_{y}$, since $\pi_{z}$ is a constant of the motion $^{18}$ and the motion along the $z$ axis is that of a free particle. The coherent states $|\alpha, \xi\rangle$ are defined as the simultaneous eigenstates of the two commuting non-Hermitian operators which annihilate the ground state ${ }^{17}$

$$
\begin{aligned}
& \pi_{-}|N=0, m=0\rangle=0, \\
& X_{+}|N=0, m=0\rangle=0,
\end{aligned}
$$

with $^{17}$

$$
X_{ \pm}=x-\frac{\pi_{y}}{m_{r} \Omega} \pm i\left(y+\frac{\pi_{x}}{m_{r} \Omega}\right),
$$

that are called orbit-center coordinate operators that step on the angular momentum $m$ and not the energy. ${ }^{17}$ We have then

$$
\begin{gathered}
\pi_{-}|\alpha, \xi\rangle=\frac{\hbar \alpha}{i l^{2}}|\alpha, \xi\rangle, \\
X_{+}|\alpha, \xi\rangle=\xi|\alpha, \xi\rangle,
\end{gathered}
$$

where the above defined quantity $l$ represents the classical radius of the ground-state's Landau orbit. Evaluating now $\left\langle\alpha, \xi\left|\pi_{+} \pi_{-}\right| \alpha, \xi\right\rangle$ we immediately find the modulus squared of eigenvalue $\alpha$ as given by

$$
|\alpha|^{2}=\frac{l^{4}}{\hbar^{2}}\left\{\left(p_{x}-\frac{\hbar y}{2 l^{2}}\right)^{2}+\left(p_{y}+\frac{\hbar x}{2 l^{2}}\right)^{2}\right\} .
$$

The terms within the curly brackets (divided by $2 m_{r}$ ) yield the classical energy $\mathcal{E}_{\text {mag }}$ of an electron in a uniform magnetic field. As noted in Ref. 17, the modulus of both $\alpha$ and $\xi$ has dimensions of length.

After expanding the states $|\alpha, \xi\rangle$ in the complete set of energy eigenfunctions $|N, m\rangle$ given above, and using Eqs. (3.4) and (3.6) of Ref. 17, we immediately obtain the probability of finding the electron in the state $|\alpha, \xi\rangle$ as

$$
|\langle N, m \mid \alpha, \xi\rangle|^{2}=\frac{|\alpha|^{2 N}|\xi|^{2(N-m)}}{\left(2 l^{2}\right)^{N} N !\left(2 l^{2}\right)^{N-m}(N-m) !} e^{-\left(|\alpha|^{2}+|\xi|^{2}\right) / 2 l^{2}} .
$$

Our coherent states $|\alpha, \xi\rangle$ satisfy the closure relation ${ }^{17}$

$$
\int \frac{\mathrm{d}^{2} \alpha \mathrm{d}^{2} \xi}{4 \pi^{2} l^{4}}|\alpha, \xi\rangle\langle\alpha, \xi|=1
$$

as expected. 


\section{HUSIMI DISTRIBUTION AND WEHRL ENTROPY}

We begin at this point our present endeavor, i.e., introducing thermodynamics into the model of the preceding section, by calculating the appropriate Husimi distribution (3) that our model requires. Such distribution adopts the appearance

$$
\mu\left(x, p_{x} ; y, p_{y}\right)=\frac{1}{Z} \sum_{N=0}^{\infty} \sum_{m=-\infty}^{N} e^{-\beta E_{N}|\langle N, m \mid \alpha, \xi\rangle|^{2}} .
$$

Using (16) one can rewrite the above expression in the fashion

$$
\begin{aligned}
\mu\left(x, p_{x} ; y, p_{y}\right)= & \frac{e^{-\beta \hbar \Omega / 2}}{Z} e^{-\left(|\alpha|^{2}+|\xi|^{2}\right) / 2 l^{2}} \sum_{N=0}^{\infty} \frac{|\alpha|^{2 N}|\xi|^{2 N} e^{-\beta \hbar \Omega N}}{\left(2 l^{2}\right)^{2 N} N !} \\
& \times \sum_{m=-\infty}^{N}\left(\frac{2 l^{2}}{|\xi|^{2}}\right)^{m} \frac{1}{(N-m) !}
\end{aligned}
$$

and pass to the evaluation of the sum

$$
\sum_{m=-\infty}^{N}\left(\frac{2 l^{2}}{|\xi|^{2}}\right)^{m} \frac{1}{(N-m) !}=\left(\frac{|\xi|^{2}}{2 l^{2}}\right)^{-N} e^{|\xi|^{2} / 2 l^{2}}
$$

This last result is now replaced into (19) so as to arrive at

$$
\mu\left(x, p_{x} ; y, p_{y}\right)=\frac{e^{-\beta \hbar \Omega / 2}}{Z} e^{-|\alpha|^{2} / 2 l^{2}} \sum_{N=0}^{\infty}\left[\frac{|\alpha|^{2}}{2 l^{2}} e^{-\beta \hbar \Omega}\right]^{N} \frac{1}{N !},
$$

which immediately leads to the desired Husimi result we were looking for (our first new result), namely,

$$
\mu\left(x, p_{x} ; y, p_{y}\right)=\frac{e^{-\beta \hbar \Omega / 2}}{Z} e^{-\left(1-e^{-\beta \hbar \Omega}\right)|\alpha|^{2} / 2 l^{2}} .
$$

The appropriate partition function $Z$ that we need here, i.e., that for a particle in a cylindrical geometry (length $L$ and radius $R$ ), oriented along the magnetic field, has been given by Feldman et al. One has $Z_{\text {perp }} Z_{\text {parall, }}$ where $Z_{\text {parall }}$ is the usual partition function for one-dimensional free motion $Z_{\text {parall }}=(L / \hbar)\left(2 \pi m_{r} k T\right)^{1 / 2} \cdot{ }^{17} Z$ has the form ${ }^{17}$

$$
Z=V \frac{\left(2 \pi m_{r} k T\right)^{1 / 2}}{h} \frac{m_{r} \Omega}{4 \pi \hbar} \frac{1}{\sinh (\beta \hbar \Omega / 2)} .
$$

Using it we can easily recast $\mu\left(x, p_{x} ; y, p_{y}\right)$ as

$$
\begin{aligned}
\mu\left(x, p_{x} ; y, p_{y}\right)= & \frac{4 \pi^{2} \hbar^{2}}{V m_{r} \Omega\left(2 \pi m_{r} k T\right)^{1 / 2}} \\
& \times\left(1-e^{-\beta \hbar \Omega}\right) e^{-\left(1-e^{-\beta \hbar \Omega}\right)|\alpha|^{2} / 2 l^{2}} .
\end{aligned}
$$

This last expression is not yet normalized [the pertinent normalization integral equals $2 \pi \hbar /\left(L \sqrt{2 \pi m_{r} k T}\right)$, with $L$ the length of the sample]. This can be remedied by scaling the above Husimi distribution. We proceed in two steps. First we define

$$
\varphi\left(x, p_{x} ; y, p_{y}\right)=\frac{V m_{r} \Omega\left(2 \pi m_{r} k T\right)^{1 / 2}}{4 \pi^{2} \hbar^{2}} \mu\left(x, p_{x} ; y, p_{y}\right)
$$

and write

$$
\varphi\left(x, p_{x} ; y, p_{y}\right)=\left(1-e^{-\beta \hbar \Omega}\right) e^{-\left(1-e^{-\beta \hbar \Omega}\right)|\alpha|^{2} / 2 l^{2}} .
$$

Although this is not yet normalized, it is dimensionless. Now the corresponding normalization integral yields $A m_{r} \Omega /(2 \pi \hbar)$. Finally, the normalized distribution is, of course,

$$
\phi\left(x, p_{x} ; y, p_{y}\right)=\frac{2 \pi \hbar}{A m_{r} \Omega}\left(1-e^{-\beta \hbar \Omega}\right) e^{-\left(1-e^{-\beta \hbar \Omega}\right)|\alpha|^{2} / 2 l^{2}} .
$$

Obviously, we write now the Wehrl entropy in terms of the distribution function $\phi\left(x, p_{x} ; y, p_{y}\right)$ and get

$$
W=-\int \frac{\mathrm{d}^{2} \alpha \mathrm{d}^{2} \xi}{4 \pi^{2} l^{4}} \phi\left(x, p_{x} ; y, p_{y}\right) \ln \phi\left(x, p_{x} ; y, p_{y}\right),
$$

so that, after replacing (27) into $W$ we find

$$
W=1-\ln \left(1-e^{-\beta \hbar \Omega}\right)-\ln \left(\frac{2 \pi l^{2}}{A}\right),
$$

where we have used the following result given in Ref. 17,

$$
\int \frac{\mathrm{d}^{2} \alpha \mathrm{d}^{2} \xi}{4 \pi^{2} l^{4}} e^{-\left(1-e^{-\beta \hbar \Omega}\right)|\alpha|^{2} / 2 l^{2}}=\frac{A \mu \Omega}{2 \pi \hbar} \frac{1}{1-e^{-\beta \hbar \Omega}} .
$$

$W$ depends on the sample's dimensions via the third term in (29). The effect of the magnetic field is reflected via $\Omega$. The important point is the following: the present Wehrl measure is, save for the above mentioned (constant) third term, identical to that of an harmonic oscillator of frequency $\Omega$ at the temperature $T .{ }^{19}$ This constitutes our second original (present) contribution. It is to be pointed out that this result confirms an hypothesis made 10 years ago in Ref. 11, whose authors conjectured that the form (29) found for the harmonic oscillator could be of a rather general character.

\section{FISHER'S INFORMATION MEASURE}

Fisher advanced, already in the 1920s, a quite interesting information measure (for a detailed study see Refs. 1 and 2). Consider a $\theta-\mathbf{z}$ "scenario" in which we deal with a system specified by a physical parameter $\theta$, while $\mathbf{z}$ is a stochastic variable $\left(\mathbf{z} \in \mathfrak{R}^{M}\right)$ and $f_{\theta}(\mathbf{z})$ the probability density for $\mathbf{z}$ (that depends also on $\theta$ ). One makes a measurement of $\mathbf{z}$ and has to best infer $\theta$ from this measurement, calling the resulting estimate $\tilde{\theta}=\tilde{\theta}(\mathbf{z})$. The question is how well $\theta$ can be determined. Estimation theory ${ }^{2}$ states that the best possible estimator $\tilde{\theta}(\mathbf{z})$, after a very large number of $\mathbf{z}$ samples is examined, suffers a mean-square error $\varepsilon^{2}$ from $\theta$ that obeys a relationship involving Fisher's $I$, namely, $I \varepsilon^{2}=1$, where the Fisher information measure $I$ is of the form

$$
I(\theta)=\int \mathrm{d} \mathbf{z} f_{\theta}(\mathbf{z})\left\{\frac{\partial \ln f_{\theta}(\mathbf{z})}{\partial \theta}\right\}^{2} .
$$

This "best" estimator is the so-called efficient estimator. Any 
other estimator exhibits a larger mean-square error. The only caveat to the above result is that all estimators be unbiased, i.e., satisfy $\langle\tilde{\theta}(\mathbf{z})\rangle=\theta$. Fisher's information measure has a lower bound: no matter what parameter of the system one chooses to measure, $I$ must be larger or equal than the inverse of the mean-square error associated with the concomitant experiment. This result,

$$
I \varepsilon^{2} \geqslant 1
$$

is referred to as the Cramer-Rao bound. ${ }^{2}$ The uncertainty principle can be regarded as a special instance of (32). ${ }^{2}$ One often speaks of "generalized" uncertainty relations.

A particular I-case is of great importance, that of translation families, ${ }^{2,3}$ i.e., distribution functions (DF) whose form does not change under $\theta$-displacements. These DF are shiftinvariant (à la Mach, no absolute origin for $\theta$ ), and for them Fisher's information measure adopts the somewhat simpler appearance $^{2}$

$$
I=\int \operatorname{dz} f(\mathbf{z})\left\{\frac{\partial \ln f(\mathbf{z})}{\partial \mathbf{z}}\right\}^{2} .
$$

Fisher's measure is additive. ${ }^{2}$ Here we deal with the issue of estimating localization in a thermal scenario that revolves around a four-dimensional phase space, i.e., $\mathbf{z}$ $\equiv\left(z_{1}, z_{2}, z_{3}, z_{4}\right)$ is a four-dimensional vector. Such an estimation task leads, as shown in Ref. 8, to the thermodynamics of the problem. Our Fisher measure acquires the appearance, ${ }^{19}$

$$
I=\sum_{i}^{4} I_{i}=\sum_{i}^{4} \int \mathrm{d} z_{i} f\left(z_{1}, z_{2}, z_{3}, z_{4}\right)\left\{\frac{\partial \ln f\left(z_{i}\right)}{\partial z_{i}}\right\}^{2} .
$$

\section{FISHER INFORMATION AND LANDAU DIAMAGNETISM}

We are now in conditions to obtain explicitly the Fisher's information measure, by using the formalism previously described. Since $\ln \phi=\ln \left(2 \pi \hbar / A m_{r} \Omega\right)+\ln \left(1-e^{-\beta \hbar \Omega}\right)$ $-\left(1-e^{-\beta \hbar \Omega}\right)|\alpha|^{2} / 2 l^{2}$, the result (15) allows for the immediate finding

$$
\begin{gathered}
\frac{\partial \ln \phi}{\partial x}=\frac{1-e^{-\beta \hbar \Omega}}{2 \hbar}\left(p_{y}+\frac{\hbar x}{2 l^{2}}\right), \\
\frac{\partial \ln \phi}{\partial y}=\frac{1-e^{-\beta \hbar \Omega}}{2 \hbar}\left(p_{x}-\frac{\hbar y}{2 l^{2}}\right), \\
\frac{\partial \ln \phi}{\partial p_{x}}=\frac{l^{2}\left(1-e^{-\beta \hbar \Omega}\right)}{\hbar^{2}}\left(p_{x}-\frac{\hbar y}{2 l^{2}}\right),
\end{gathered}
$$

and

$$
\frac{\partial \ln \phi}{\partial p_{y}}=\frac{l^{2}\left(1-e^{-\beta \hbar \Omega}\right)}{\hbar^{2}}\left(p_{y}+\frac{\hbar x}{2 l^{2}}\right) .
$$

With the above expressions we can now recast (15) in the fashion

$$
|\alpha|^{2}=\frac{2 l^{4}}{\left(1-e^{-\beta \hbar \Omega}\right)^{2}} \mathcal{A},
$$

where

$$
\mathcal{A}=\left(\frac{\partial \ln \phi}{\partial x}\right)^{2}+\left(\frac{\partial \ln \phi}{\partial y}\right)^{2}+\frac{\hbar^{2}}{4 l^{4}}\left[\left(\frac{\partial \ln \phi}{\partial p_{x}}\right)^{2}+\left(\frac{\partial \ln \phi}{\partial p_{y}}\right)^{2}\right] \text {. }
$$

We are now in a position to write down the Fisher measure by following the prescription (34) (Refs. 19 and 20) and then write

$$
I=\int \frac{\mathrm{d}^{2} \alpha \mathrm{d}^{2} \xi}{4 \pi^{2} l^{4}} \phi\left(x, p_{x} ; y, p_{y}\right) l^{2} \mathcal{A},
$$

which, after a little algebra, turns out to be

$$
I=\frac{\left(1-e^{-\beta \hbar \Omega}\right)^{2}}{2 l^{2}} \int \frac{\mathrm{d}^{2} \alpha \mathrm{d}^{2} \xi}{4 \pi^{2} l^{4}}|\alpha|^{2} \phi\left(x, p_{x} ; y, p_{y}\right) .
$$

The integration is performed by appropriately using the pertinent derivatives of (30). We finally obtain

$$
I=1-e^{-\beta \hbar \Omega} \text {. }
$$

A glance at Ref. 19 tells us that the above is just the Fisher measure for the harmonic oscillator, which constitutes our third original result. No dependence on the sample's size parameters is detected. This is a direct result of the infinite degeneracy discussed above, which entails that estimating position in phase space loses physical relevance.

We can finally compare the information (43) with the Wehrl measure (29), concluding that

$$
W=1-\ln I-\ln \left(\frac{2 \pi l^{2}}{A}\right),
$$

i.e., they are complementary informational quantities. ${ }^{19}$ As a matter of fact, we establish here one of the few existing direct Shannon-Fisher links. Since we already know that $W$ $\geqslant 1$, this leads to the inequality

$$
I \leqslant A /\left(2 \pi l^{2}\right),
$$

still another original result. When the area of the sample is microscopic, $A=2 \pi l^{2}$, the inequality reduces to that characterizing the harmonic oscillator, i.e., $I \leqslant 1$.

For didactic reasons it is now convenient to focus attention on the quantity $|\alpha|^{2}=2 m_{r}\left(l^{4} / \hbar^{2}\right) \mathcal{E}_{\text {mag }}$, the "natural variable" of our scenario. Going back to Eq. (42), we notice that the integral there is just $\left\langle|\alpha|^{2}\right\rangle$, i.e., proportional to the semiclassical mean magnetic energy $\left\langle\mathcal{E}_{\text {mag }}\right\rangle$ [see the comment that follows Eq. (15)]. In other words, estimating localization in phase space is for the present problem equivalent to evaluating the average energy of our electron. It is pertinent to ask now about $|\alpha|$ - fluctuations. A quick calculation yields

$$
\langle|\alpha|\rangle^{2}=\frac{\pi l^{2}}{2 I}
$$

and 


$$
(\Delta\langle|\alpha|\rangle)^{2}=\left\langle|\alpha|^{2}\right\rangle-\langle|\alpha|\rangle^{2}=\frac{4-\pi}{2} \frac{l^{2}}{I} .
$$

Our phase space localization problem becomes intimately linked to these fluctuations. The ensuing $(\Delta\langle|\alpha|\rangle)^{2} I$-product, i.e., the $|\alpha|$-Cramer-Rao bound (32) (generalized uncertainty principle ${ }^{2}$ ) is

$$
(\Delta\langle|\alpha|\rangle)^{2} I=\frac{4-\pi}{2} l^{2}=\frac{4-\pi}{2} \frac{c}{e H} \hbar,
$$

and we observe (i) as an equal sign is obtained, the estimation is optimal in the sense that the lower bound of the inequality (32) is always obtained, ${ }^{2}$ (ii) the associated uncertainty is independent of the temperature, and (iii) as we increase localization-quality ( $I$ increases), the size of $|\alpha|$-fluctuations, reasonably enough, decreases. A controlparameter, namely, the magnetic field intensity $H$, is available. The larger the intensity, the better the overall quality. Nature imposes the ultimate control, however, as given by $\hbar$. The difference between $(4-\pi) / 2$ and $1 / 2$ (of the order of $10^{-1}$ ) is due to the semiclassical character of our treatment. We look now for a Cramer-Rao inequality that directly involves the energy $\mathcal{E}_{\text {mag. }}$. Things will drastically change because to get the energy from $|\alpha|^{2}$ one must divide by $l^{4}$, which in turn will reverse the $H$ role. We immediately find

$$
\left\langle\mathcal{E}_{\text {mag }}\right\rangle=\frac{\hbar \Omega}{I}
$$

and

$$
\left\langle\mathcal{E}_{\text {mag }}^{2}\right\rangle=2 \frac{\hbar^{2} \Omega^{2}}{I^{2}},
$$

so that for the energy-fluctuation $\Delta^{2} \mathcal{E}_{\text {mag }}=\left\langle\mathcal{E}_{\text {mag }}^{2}\right\rangle-\left\langle\mathcal{E}_{\text {mag }}\right\rangle^{2}$ one finds

$$
\Delta \mathcal{E}_{\mathrm{mag}} I=\hbar \Omega=\hbar \frac{e H}{m_{r} c},
$$

which, once again, is independent of $T$. The effect of $H$ is clearly different now, as anticipated. It is a simple matter to verify that (51) also gives a "localization-energy vs fluctuations" Cramer-Rao uncertainty identical to the one that obtains for the harmonic oscillator. The smaller the energy fluctuations, the better the localization estimation via $I$. Since the infinite degeneracy of the problem does not affect energy considerations, it is understandable that the result (51) be identical to the one that holds for the harmonic oscillator.

\section{CONCLUDING REMARKS}

We have here focused attention upon Landau's diamagnetism. A semiclassical information theory undertaking was tackled, (i) trying to estimate phase-space location via Fisher information and (ii) evaluating the semiclassical Wehrl entropy. As a summary

(i) Using the coherent states discussed in Ref. 17 we have explicitly given the form of the Husimi distribution function for a spinless electron in a uniform magnetic field [cf. Eq. (22)].

(ii) We have discovered that the Wehrl entropy for Landau's diamagnetism is, save for a constant term that depends on the size of the sample, that of a thermal harmonic oscillator whose frequency is the cyclotron one.

(iii) For the corresponding Fisher measure the above similitude becomes identity. The thermostatistics of the two problems is thus the same at the semiclassical level. This is of course a defect, that can be traced to the infinite degeneracy of the system's energy levels.

(iv) We confirmed a conjecture made in Ref. 11, in the sense that the form (29) is indeed of a rather general character.

(v) An uncertainty relation linking phase space localization with energy fluctuations has been discovered [cf. Eq. (51)].

\section{ACKNOWLEDGMENT}

One of us (S.C) would like to thank partial financial support by FONDECYT, Grant No. 1010776.
${ }^{1}$ B. R. Frieden and B. H. Soffer, Phys. Rev. E 52, 2274 (1995).

${ }^{2}$ B. R. Frieden, Physics from Fisher Information (Cambridge University Press, Cambridge, England, 1998).

${ }^{3}$ F. Pennini, A. R. Plastino, and A. Plastino, Physica A 258, 446 (1998).

${ }^{4}$ B. R. Frieden, A. Plastino, A. R. Plastino, and H. Soffer, Phys. Rev. E 60, 48 (1999).

${ }^{5}$ F. Pennini, A. Plastino, A. R. Plastino, and M. Casas, Phys. Lett. A 302, 156 (2002).

${ }^{6}$ A. R. Plastino and A. Plastino, Phys. Rev. E 54, 4423 (1996).

${ }^{7}$ A. Plastino, A. R. Plastino, and H. G. Miller, Phys. Lett. A 235, 129 (1997)

${ }^{8}$ R. Frieden, A. Plastino, A. R. Plastino, and B. H. Soffer, Phys. Rev. E 60, 48 (1999); 66, 046128 (2002); Phys. Lett. A 304, 73 (2002); S. Flego, R. Frieden, A. Plastino, A. R. Plastino, and B.
H. Soffer, Phys. Rev. E 68, 016105 (2003).

${ }^{9}$ A. Wehrl, Rep. Math. Phys. 16, 353 (1979).

${ }^{10}$ S. Gnuzmann and K. Życzkowski, J. Phys. A 34, 10123 (2001).

${ }^{11}$ A. Anderson and J. J. Halliwell, Phys. Rev. D 48, 2753 (1993).

${ }^{12}$ R. J. Glauber, Phys. Rev. 131, 2766 (1963).

${ }^{13}$ J. R. Klauder and B. S. Skagerstam, Coherent States (World Scientific, Singapore, 1985).

${ }^{14}$ J. Schnack, Europhys. Lett. 45, 647 (1999).

${ }^{15}$ K. Husimi, Proc. Phys. Math. Soc. Jpn. 22, 264 (1940).

${ }^{16}$ E. H. Lieb, Commun. Math. Phys. 62, 35 (1978).

${ }^{17}$ A. Feldman and A. H. Kahn, Phys. Rev. B 1, 4584 (1970).

${ }^{18}$ M. H. Johnson and B. A. Lippmann, Phys. Rev. 76, 828 (1949).

${ }^{19}$ F. Pennini and A. Plastino, Phys. Rev. E 69, 057101 (2004).

${ }^{20}$ F. Pennini and A. Plastino, Phys. Lett. A 326, 20 (2004). 